\title{
Utilización y gasto en servicios de salud de los individuos en Argentina en 2005. Comparaciones internacionales de diferenciales socio-económicos en salud
}

\author{
Use of and spending in health services by individuals in \\ Argentina in 2005. International comparisons of \\ socio-economic differentials in health
}

Jorge Raúl Jorrat ${ }^{1}$

María de las Mercedes Fernández²

Elida H. Marconi ${ }^{3}$

\footnotetext{
${ }^{1}$ Contador Público Nacional, Universidad Nacional de Tucumán. Doctor en Sociología, Universidad Estatal de Michigan. Director del Centro de Estudios de Opinión Pública, Instituto G. Germani, Universidad de Buenos Aires. Investigador del CONICET. jrjorrat@mail.fsoc.uba.ar

${ }^{2}$ Licenciada en Sociología, Universidad de Buenos Aires. Coordinadora del área de Análisis y Difusión, Dirección de Estadística e Información de Salud (DEIS), Ministerio de Salud de la Nación (MSN). mfernandez@deis.gov.ar

${ }^{3}$ Licenciada en Sociología, Universidad de Buenos Aires. Directora de la Dirección de Estadística e Información de Salud (DEIS), Ministerio de Salud de la Nación (MSN). emarconi@deis.gov.ar
}

RESUMEN Este trabajo, continuidad de un relevamiento nacional anterior (2003), intenta actualizar las pautas de utilización y gasto en servicios de salud explorando, como en el pasado, las bases sociales de tales comportamientos. La indagación contempló el interés de políticas públicas en este sentido.

Los resultados considerados, surgidos de una muestra nacional a 1.546 personas de 0 años y más, muestran que -dentro de lo esperado- el gasto en salud es relevante dentro del gasto total de los hogares y que el gasto de bolsillo en medicamentos es una proporción muy importante $(40 \%)$ de ese gasto en salud.

Como parte sustantiva del artículo, se agrega un apartado especial referido a aspectos de desigualdad en salud. La posibilidad de comparar resultados locales tomando como parámetro Japón resulta de indudable interés para evaluar los diferenciales socioeconómicos en salud. La metodología básica es similar en ambos estudios, descansando en ecuaciones de regresión logística.

PALABRAS CLAVE Atención a la Salud; Desigualdades en la Salud; Gastos en Salud; Financiación en Salud; Políticas Públicas.

\begin{abstract}
This paper, a follow-up to a previous national survey (2003), intends to update the health care services use and expenditure patterns by exploring, as in the past, the social bases for such behaviors. The survey included public policy interest in this regard.

The results analyzed, from a national population sample of 1.546 persons aged 0 and older, show that -as expected- health expenditure is significant within total household expenditure and that out-of-pocket-expenses in medication are a very important proportion (40\%) of this health expenditure.

As a fundamental part of this article, there is a special section on health inequality aspects. The possibility of comparing local results using Japan as a parameter is of unquestionable interest to evaluate socioeconomic differentials in this field. The basic method is similar in both studies, relying on logistic regression equations.
\end{abstract}

KEY WORDS Health Care (Public Health); Health Inequalities; Health Expenditures; Financing, Health; Public Policies. 


\section{INTRODUCCIÓN}

En 2005 se releva una "Encuesta de Utilización y Gasto en Servicios de Salud", que ofrece una nueva mirada sobre la utilización y gasto en servicios de salud para el país luego del primer estudio de alcance nacional del año 2003 (1), permitiendo explorar en qué medida el comportamiento sobre utilización y gasto en servicios de salud ha variado al aumentar la distancia temporal respecto de la crisis de fines de 2001, cuyos efectos podrían haberse reflejado en los resultados de 2003 (a).

Los objetivos de dicho relevamiento pueden resumirse en los siguientes puntos:

$\rightarrow$ Constituirse en el segundo estudio nacional sobre utilización y gasto en servicios de salud por parte de la población.

$\rightarrow$ Permitir una exploración de las bases sociales de la utilización y el gasto en servicios de salud a partir de una amplia información sociodemográfica para cada encuestado.

$\rightarrow$ Explorar, adicionalmente, la asociación entre condiciones de salud y variables sociodemográficas, indagando sobre diferenciales socioeconómicos en salud, dentro de una perspectiva comparativa.

Es necesario enfatizar que las encuestas de utilización y gasto en servicios de salud 2003 y 2005 son el único relevamiento estadístico, dentro de la metodología de encuestas a hogares o a población, que posibilita analizar en una misma unidad-conceptual y operativamente- la cobertura, la utilización y el gasto directo en salud de la población. Hasta donde llega nuestra información, otras encuestas o censos nacionales solo toman uno de estos aspectos (o utilización, o gasto, o cobertura).

Nuestro acento está más en explorar las bases sociales de la utilización y gasto en salud que en estimaciones precisas del gasto, atento a la demanda de mayores datos que esto implica $(2,3)$. La indagación pretende estar más cerca de una "sociología de la salud" -o "en la salud" $(4,5)-$ que de una "economía de la salud". Y siempre dentro de preocupaciones por considerar variables sociológicas en estos estudios, sintetizadas, por ejemplo, por Donald W. Ligth (6).

\section{ALGUNOS ASPECTOS CONCEPTUALES}

El eje teórico que presupone la encuesta es que existen desigualdades con relación a la cobertura, la utilización y el gasto en salud, según distintas variables sociodemográficas: edad, sexo, nivel de instrucción, ocupación (clase social), nivel de ingreso y localización espacial de la población.

$\rightarrow$ Los factores socioeconómicos interrelacionados entre sí, inciden en la utilización de los servicios, estableciendo diferencias entre los distintos grupos o sectores sociales. La vinculación entre variables sociodemográficas y el comportamiento en salud es generalmente aceptada, aunque la magnitud y forma de esta asociación depende de las aproximaciones metodológicas y de la operacionalización de las variables en juego. Una forma más fuerte de decirlo: "La salud, o la percepción del estado de salud, no se distribuye en la sociedad de una forma aleatoria. Las variables sociográficas y socioeconómicas hacen que la salud se distribuya de acuerdo a ciertas leyes" (7 p.64).

$\rightarrow$ La condición de afiliación (b) y el gasto de bolsillo en salud inciden en el presupuesto familiar de manera distinta según los grupos sociales. Los antecedentes existentes muestran que el gasto en salud es relevante dentro del gasto total directo de los hogares, y, dentro de éste, el gasto en medicamentos da cuenta de una parte muy importante del gasto en salud.

Debe notarse que, si bien pueden encontrarse coincidencias con respecto a los mencionados aspectos conceptuales generales sobre desigualdad en salud y enfermedad, de lo que se trata es de avanzar en la especificación y medición de las interrelaciones y efectos emergentes. El presente estudio es un ejercicio en esa dirección, dentro de los límites de esta propuesta que más bien enfatiza los aspectos exploratorio-descriptivos. 
Por otro lado, este artículo, además de ofrecer una presentación sucinta de esos aspectos, cede espacio a una indagación comparativa internacional sobre diferenciales sociales en salud, a partir de un estudio semejante realizado para Japón por Hiroshi Ishida (8).

Una parte de la bibliografía sobre desigualdad en salud se propone distinguir "desigualdad" de "inequidad" $(4,5,9,10)$ mientras que otros discuten aspectos problemáticos de la distinción pobreza-igualdad-equidad, notando que la distinción muchas veces carece de ventajas prácticas o tiene un interés académico, sin negar su utilidad (11).

El tema puede dar lugar a una amplia discusión, siendo suficiente señalar aquí la necesidad de explorar empíricamente las desigualdades en salud, evitando entrar en posibles juicios normativos. Para este objetivo, se puntualiza (10) que la bibliografía señala como una de dos alternativas amplias de evaluación de la desigualdad en salud, la más común, a la medición de diferencias de grupos sociales en salud, que es lo que se intentará en este estudio. También señalan estos autores que hay preocupación respecto de las comparaciones internacionales, por las diferencias de comparabilidad de grupos relevantes o variaciones en su composición social en el tiempo. [Precauciones, para aspectos de auto-percepción de salud, pueden encontrarse en Zimmer Z, et al. (2)]. Una ventaja para enfrentar estas preocupaciones es la posibilidad de contar, cada vez más, con encuestas con un alto grado de estandarización internacional. En este sentido, la encuesta en que descansó el estudio de Japón se aproxima bastante a la encuesta utilizada localmente, para las variables de interés, lo que brinda una oportunidad poco común para evaluaciones comparativas en nuestro medio. Más aun, como señala Donald Acheson (12 p.1):

Si bien es claramente inapropiado generalizar a partir de la experiencia de un país en términos de políticas particulares, el peso de la evidencia científica sugiere, [...] que una explicación socioeconómica de las desigualdades en salud es pasible de resultar relevante para todos los países ricos y pobres del mundo.

\section{DATOS, VARIABLES, METODOLOGÍA}

Los datos usados provienen de una encuesta nacional domiciliaria relevada en 2005 -1.546 casos de 0 años y más-, a partir de un cuestionario altamente estructurado que, básicamente, repite preguntas de un relevamiento de 2003 (c). Las variables de utilización y gasto están repetidamente descriptas en las distintas publicaciones de la Dirección de Estadísticas e Información en Salud del Ministerio de Salud de la Nación (DEIS-MSN), a las que pueden consultarse, si bien su interpretación directa es razonablemente clara (13).

Las variables vinculadas a la evaluación comparativa con Japón se refieren a condiciones de salud. De las cinco condiciones de salud -variables dependientes- tomadas en cuenta por Ishida, solo cuatro fueron consideradas en la encuesta local (d). La primera hace referencia a la presencia de enfermedades crónicas diagnosticadas por un médico. La segunda condición de salud fue la de consulta al médico (incluyendo en el caso japonés la visita al dentista) en el último año. La tercera condición de salud considerada por Ishida fue la presencia de dolor o malestar físico, lo que no fue tomado en cuenta en el caso local. La cuarta condición de salud se refiere a las habilidades físicas del entrevistado. A los encuestados se los interrogó sobre si tuvieron que interrumpir actividades como ir al trabajo, hacer tareas del hogar o ir a un establecimiento educativo. Finalmente, la quinta variable se refiere a autopercepción de la propia salud. Las peores condiciones se codificaban 1 , las mejores 0 . Con el objeto de mostrar que si bien las variables dependientes están interrelacionadas pero que miden condiciones diferentes, Ishida da cuenta de que las correlaciones entre ellas "no son excepcionalmente altas". Los resultados en ambos países favorecen la consideración de cada una de estas condiciones (tales resultados no se presentan aquí). Como la muestra usada por Ishida se refiere a personas de 20 años y más, en esta parte del estudio el análisis de datos locales se circunscribirá a ese segmento de edad (20 años y más).

Por lo que respecta a las variables sociodemográficas consideradas en el estudio japonés, clase social se categoriza en ambos 
casos según propuesta conocida como Erikson, Goldthorpe y Portocarero (EGP) (14), elaborado en distintas oportunidades, por ejemplo por Erikson R, Goldthorpe JH (15), y puede encontrarse una construcción local en Jorrat JR, (16). Ishida usa una clasificación de EGP en seis categorías, que se respetan para los datos de Argentina:

1) Clase de servicios. Comprende a los profesionales de nivel alto y bajo, administradores y gerentes.

2) Empleados no manuales rutinarios. Comprenden posiciones con relaciones de empleo mezcladas, en administración, comercio, personal de ventas, etc.

3) Pequeña burguesía. Abarca a los pequeños propietarios y artesanos con empleados y a aquellos sin empleados, fuera del trabajo rural.

4) Trabajo rural. Ídem anterior, incluyendo el trabajo manual rural.

5) Trabajadores manuales calificados. Tienen claramente un contrato de trabajo. Incluye supervisores de trabajadores manuales y técnicos de nivel bajo, además de los obreros manuales calificados.

6) Trabajadores manuales no calificados. Incluye obreros manuales semi y no calificados, en la producción general, excluido rural.

Siguiendo a este autor, se utilizará la categoría de clase más alta (clase de servicios) como grupo de referencia de base en la regresión logística (no entrará en la regresión). En ambos casos, educación está medida en años de educación completados. Como en el estudio de Japón, edad (en años cumplidos) y género (varón $=1$, mujer $=0$ ) se incluyen como variables de control. Además, se incluye el tamaño de las ciudades donde vive el entrevistado: para el caso local, grandes (500 mil habitantes y más), medianas (de $100 \mathrm{mil}$ a menos de $500 \mathrm{mil}$ ) y pequeñas/rurales (menos de 100 mil habitantes), quedando las pequeñas localidades y las zonas rurales como categoría de referencia de base. Finalmente, ingreso se define para Japón como el ingreso familiar total anual (en millones de yenes), mientras que en Argentina se anualiza el promedio de ingreso mensual per capita declarado en la encuesta. Dada la alta presencia de no respuesta de ingreso en la encuesta manejada por Ishida -lo que también ocurre a nivel local-, este autor introduce una variable dicotómica $(=1$ si no declaraba, $=0$ en otro caso) para representar a dichos no respondentes, procedimiento que seguiremos en la comparación.

En la primera parte, más breve, se descansa en el cálculo de porcentajes de utilización de servicios de salud según segmentos sociodemográficos y en promedios de gastos en esos distintos servicios según los mismos segmentos (e).

En la segunda parte, distintos modelos de regresión logística binaria, para las distintas condiciones de salud, son considerados por Ishida, lo que se repetirá en la presente comparación. Nótese que en general la dicotomización de las variables implicaba asignar 1 a la peor condición, 0 a la mejor. El procedimiento de categorizar las variables dependientes como variables 0-1 es necesario para la utilización de modelos de regresión logística binaria. Ésta se usa cuando la variable dependiente es dicotómica y las independientes son de cualquier tipo. La regresión logística transforma la variable dependiente en una variable denominada logit, basada en el logaritmo natural de las chances (odds) de la ocurrencia de la variable dependiente. Así, la regresión logística estima la probabilidad de ocurrencia de un cierto evento. Para facilitar la lectura, se toma en cuenta básicamente la presencia significativa -o no- de los coeficientes de regresión de las variables independientes. Nótese que:

Los coeficientes de regresión logística simplemente muestran el cambio en la predicción del logaritmo de las chances de experimentar un evento o de tener una característica dado el cambio de una unidad en las variables independientes (18 p.19).

Una observación final para concluir este punto. Al comparar las distintas ecuaciones de regresión para Argentina y Japón, en algunas ecuaciones de Japón se introduce como variable independiente "Activos del hogar", que no era de interés en nuestro relevamiento. Se dudó en usar un sustituto, atento a la observación de la no significación estadística de esta variable en todas las ecuaciones presentadas salvo algunas en el Cuadro 7, lo que llevó en principio a considerarla redundante. 
No es extraño que ello sea así, porque activos del hogar entra en la ecuación junto con ingresos y se puede presumir una importante correlación positiva entre ellas, sugiriendo posibles problemas de multicolinealidad (altas correlaciones entre las variables independientes que pueden afectar las estimaciones). Finalmente se trabajó con una alternativa, cual es la auto-percepción de posición social relativa, en una escala de 1 a 10 . Mientras en el caso japonés se trataría de "riqueza objetiva", en nuestro caso se referiría a "percepción de riqueza".

\section{RESULTADOS Y DISCUSIÓN}

A continuación se ofrecen resultados y su discusión para los tres puntos básicos que abarca el artículo: a) utilización de servicios de salud, b) gasto en dichos servicios y una primera evaluación de desigualdad en salud según quintiles de ingreso y, finalmente, c) condiciones de salud y diferenciales socio-económicos en salud.

\subsection{UTILIZACIÓN DE SERVICIOS}

En primer lugar, se presenta una síntesis del comportamiento en salud de los argentinos. La estructura básica de la utilización de servicios de salud, según porcentaje de usuarios, se habría mantenido en el último bienio. En general, se nota un leve aumento, siendo lo más importante que las personas afiliadas habrían crecido un $8 \%$.

Como puede verse en el Cuadro 1, seis de cada diez personas están afiliadas, cuatro de cada diez consultaron al médico, menos de dos al dentista y algo más de dos tuvieron análisis, tratamientos o internaciones. Los usuarios de medicamentos abarcan a casi siete de cada diez encuestados.

Al considerar la utilización según segmentos sociodemográficos en 2005, la afiliación es algo mayor entre las mujeres, creciendo al aumentar la edad, el nivel de educación y el nivel de clase social, con menor presencia en las ciudades intermedias.

Por lo general, hay cierto predominio de mujeres en las distintas consultas. Mientras los consultantes al médico predominan en los extremos de grupos de edad, los consultantes al dentista o por salud mental están más presentes en las edades relativamente activas (15-64 años) y los consultantes al fonoaudiólogo disminuyen en la edad mayor. El uso de medicamentos y los análisis, tratamientos e internaciones crecen con la edad. Todos los consultantes, usuarios de medicamentos (menos claro) y los involucrados en análisis, tratamientos e internaciones tienden a aumentar su presencia con la mayor educación.

Fuera de los usuarios de medicamentos -que casi no exhiben diferencias por clase social-, la utilización de servicios de salud es mayor en las clases más acomodadas. En cuanto al nivel de ingresos, en general los usuarios predominan en los quintiles más altos de ingreso per capita familiar. Hay que notar que los consumidores de medicamentos son solo un poco más relevantes en el sector más alto de ingresos, mientras que los consultantes al médico tienen mayor presencia en los quintiles más bajos respecto del más alto. $\mathrm{O}$ sea: la consulta médica constituye el único tipo de utilización que exhibe una presencia porcentual ligeramente mayor en los sectores más pobres.

\subsection{EL GASTO EN SALUD}

En el contexto del presente relevamiento, los argentinos gastaron de bolsillo en 2005 un promedio mensual de $\$ 37$ pesos (que baja a $\$ 29$ pesos si no se tiene en cuenta al gasto en afiliación a algún sistema). Este gasto promedio mensual de toda la población varía según tipo de servicio: a medicamentos se destinan cerca de $\$ 15$ pesos, mientras que la consulta médica da cuenta de menos de $\$ 2,50$ pesos.

En el Cuadro 2, puede apreciarse que gastan más en salud las mujeres, las personas de mayor edad, los sectores de mayores recursos y mayor educación formal, los afiliados y los que viven en concentraciones urbanas importantes.

Vista en términos porcentuales, la participación diferencial del tipo de servicio de salud es relevante: el gasto poblacional promedio en medicamentos es responsable de un $40 \%$ del gasto total en salud, seguido por afiliación voluntaria (más de $21 \%$ ) y a la distancia por el 
CUadro 1. USUARIOS DE DISTINTOS SERVICIOS DE SALUD, SEGÚN VARIABLES SOCIODEMOGRÁFICAS (EN \%). POBLACIÓN DE 0 AÑOS Y MÁS. ARGENTINA, 2005.

\begin{tabular}{|c|c|c|c|c|c|c|c|}
\hline $\begin{array}{l}\text { SEGMENTOS } \\
\text { SOCIODEMOGRÁFICOS }\end{array}$ & $\begin{array}{c}\text { GASTO } \\
\text { AFILIACIÓN } \\
\text { PER CAPITA }\end{array}$ & Médico & $\begin{array}{c}\text { CONS } \\
\text { Dentista }\end{array}$ & $\begin{array}{l}\text { TAS A: } \\
\text { Psicólogo }\end{array}$ & Fonoaudiólogo & $\begin{array}{c}\text { USÓ } \\
\text { MEDICAMENTOS }\end{array}$ & $\begin{array}{l}\text { ANÁLISIS, } \\
\text { TRATAMIENTOS, } \\
\text { INTERNACIONES }\end{array}$ \\
\hline TOTAL & $62,7 \%$ & $40,6 \%$ & $15,3 \%$ & $4,1 \%$ & $2,7 \%$ & $69,2 \%$ & $22,1 \%$ \\
\hline \multicolumn{8}{|l|}{ SEXO } \\
\hline Varones & $61,5 \%$ & $35,7 \%$ & $14,0 \%$ & $3,9 \%$ & $2,5 \%$ & $69,0 \%$ & $18,3 \%$ \\
\hline Mujeres & $63,9 \%$ & $45,6 \%$ & $16,8 \%$ & $4,4 \%$ & $2,9 \%$ & $69,4 \%$ & $26,0 \%$ \\
\hline \multicolumn{8}{|l|}{ EDAD } \\
\hline 0-14 años & $57,3 \%$ & $49,5 \%$ & $11,9 \%$ & $3,1 \%$ & $2,9 \%$ & $59,4 \%$ & $14,8 \%$ \\
\hline 15-64 años & $62,8 \%$ & $35,7 \%$ & $17,5 \%$ & $4,7 \%$ & $2,8 \%$ & $71,7 \%$ & $23,5 \%$ \\
\hline 65 años y más & $84,4 \%$ & $46,0 \%$ & $10,7 \%$ & $3,2 \%$ & $1,2 \%$ & $89,0 \%$ & $40,7 \%$ \\
\hline \multicolumn{8}{|c|}{ EDUCACIÓN (18 años y más) } \\
\hline Hasta primaria completa & $52,2 \%$ & $37,0 \%$ & $11,7 \%$ & $2,0 \%$ & $1,2 \%$ & $67,2 \%$ & $20,3 \%$ \\
\hline Estudios secundarios & $61,1 \%$ & $38,6 \%$ & $14,6 \%$ & $3,5 \%$ & $2,3 \%$ & $67,8 \%$ & $19,7 \%$ \\
\hline Estudios superiores & $80,0 \%$ & $48,7 \%$ & $21,8 \%$ & $8,1 \%$ & $5,4 \%$ & $74,2 \%$ & $28,4 \%$ \\
\hline \multicolumn{8}{|l|}{ CLASE SOCIAL (EGP) } \\
\hline $\begin{array}{l}\text { Clase de servicios } \\
\text { (profesional / gerencial) }\end{array}$ & $87,4 \%$ & $44,2 \%$ & $18,9 \%$ & $7,1 \%$ & $3,3 \%$ & $69,4 \%$ & $31,7 \%$ \\
\hline $\begin{array}{l}\text { Empleados no } \\
\text { manuales rutinarios }\end{array}$ & $81,0 \%$ & $49,6 \%$ & $19,4 \%$ & $7,0 \%$ & $5,1 \%$ & $67,8 \%$ & $22,3 \%$ \\
\hline $\begin{array}{l}\text { Pequeña burguesía } \\
\text { (pequeño empleador) }\end{array}$ & $43,8 \%$ & $35,8 \%$ & $10,7 \%$ & $3,3 \%$ & $2,6 \%$ & $71,7 \%$ & $20,6 \%$ \\
\hline $\begin{array}{l}\text { Trabajadores manuales } \\
\text { calificados }\end{array}$ & $57,5 \%$ & $34,7 \%$ & $12,5 \%$ & $2,9 \%$ & $0,7 \%$ & $69,9 \%$ & $19,7 \%$ \\
\hline $\begin{array}{l}\text { Trabajadores manuales } \\
\text { no calificados }\end{array}$ & $46,9 \%$ & $38,3 \%$ & $12,9 \%$ & $0,9 \%$ & $2,8 \%$ & $68,2 \%$ & $17,3 \%$ \\
\hline $\begin{array}{l}\text { Sin ocupación / } \\
\text { no especificada }\end{array}$ & $65,0 \%$ & $40,2 \%$ & $22,0 \%$ & $5,0 \%$ & - & $69,2 \%$ & $32,0 \%$ \\
\hline \multicolumn{8}{|l|}{$\begin{array}{l}\text { QUINTIL INGRESO } \\
\text { PER CAPITA (3 grupos) }\end{array}$} \\
\hline $1+2$ & $42,8 \%$ & $44,1 \%$ & $12,3 \%$ & $2,5 \%$ & $1,3 \%$ & $70,4 \%$ & $23,0 \%$ \\
\hline $3+4$ & $74,1 \%$ & $44,2 \%$ & $16,0 \%$ & $4,0 \%$ & $2,5 \%$ & $66,5 \%$ & $19,6 \%$ \\
\hline 5 & $82,9 \%$ & $39,6 \%$ & $21,8 \%$ & $7,9 \%$ & $6,4 \%$ & $79,6 \%$ & $27,4 \%$ \\
\hline Sin especificar & $66,5 \%$ & $30,8 \%$ & $15,1 \%$ & $4,5 \%$ & $3,0 \%$ & $62,9 \%$ & $20,5 \%$ \\
\hline \multicolumn{8}{|c|}{ TAMAÑO DE LA LOCALIDAD } \\
\hline Grande & $62,6 \%$ & $40,2 \%$ & $15,7 \%$ & $6,3 \%$ & $2,0 \%$ & $66,7 \%$ & $21,9 \%$ \\
\hline Mediana & $57,0 \%$ & $39,8 \%$ & $20,2 \%$ & $1,5 \%$ & $3,7 \%$ & $70,9 \%$ & $23,0 \%$ \\
\hline Pequeña / Rural & $63,7 \%$ & $41,0 \%$ & $14,2 \%$ & $2,2 \%$ & $3,2 \%$ & $71,6 \%$ & $22,1 \%$ \\
\hline \multicolumn{8}{|l|}{ AFILIACIÓN } \\
\hline Sí & - & $43,8 \%$ & $16,5 \%$ & $5,3 \%$ & $3,0 \%$ & $69,3 \%$ & $23,8 \%$ \\
\hline No & - & $35,2 \%$ & $13,4 \%$ & $2,1 \%$ & $2,2 \%$ & $69,1 \%$ & $19,1 \%$ \\
\hline
\end{tabular}


CUadro 2. PROMEDIO DE GASTO EN SALUD DE LA POBLACIÓN, POR VARIABLES SOCIODEMOGRÁFICAS (EN PESOS). POBLACIÓN DE O AÑOS Y MÁS. ARGENTINA, 2005.

\begin{tabular}{|c|c|c|c|c|c|c|c|c|c|}
\hline $\begin{array}{l}\text { SEGMENTOS } \\
\text { SOCIODEMOGRÁFICOS }\end{array}$ & $\begin{array}{c}\text { GASTO } \\
\text { AFILIACIÓN } \\
\text { PER CAPITA }\end{array}$ & $\begin{array}{l}\text { GAS } \\
\text { Médico }\end{array}$ & $\begin{array}{l}\text { EN CONSU } \\
\text { Dentista }\end{array}$ & $\begin{array}{l}\text { TAS A: } \\
\text { Otros } \\
\text { Profesionales }\end{array}$ & MEDICAMENTOS & $\begin{array}{c}\text { ANÁLISIS, } \\
\text { TRATAMIENTOS, } \\
\text { INTERNACIONES }\end{array}$ & $\begin{array}{l}\text { OTROS } \\
\text { GASTOS }\end{array}$ & $\begin{array}{c}\text { TOTAL } \\
\text { GASTO SIN } \\
\text { AFILIACIÓN }\end{array}$ & $\begin{array}{l}\text { TOTAL } \\
\text { GENERAL } \\
\text { GASTO }\end{array}$ \\
\hline TOTAL & 7,97 & 2,44 & 3,14 & 2,58 & 14,82 & 3,23 & 3,09 & 29,30 & 37,27 \\
\hline \multicolumn{10}{|l|}{ SEXO } \\
\hline Varones & 9,39 & 1,89 & 1,88 & 2,80 & 11,86 & 4,54 & 2,40 & 25,37 & 34,75 \\
\hline Mujeres & 6,49 & 3,02 & 4,46 & 2,35 & 17,91 & 1,85 & 3,82 & 33,41 & 39,90 \\
\hline \multicolumn{10}{|l|}{ EDAD } \\
\hline 0-14 años & 2,06 & 1,86 & 2,57 & 1,64 & 11,94 & 1,46 & 2,05 & 21,51 & 23,57 \\
\hline 15-64 años & 9,84 & 2,62 & 3,42 & 3,19 & 14,33 & 2,11 & 3,81 & 29,49 & 39,33 \\
\hline 65 años y más & 16,64 & 3,31 & 3,04 & 1,19 & 31,69 & 20,92 & 1,10 & 61,26 & 77,89 \\
\hline \multicolumn{10}{|c|}{ EDUCACIÓN (18 años y más) } \\
\hline Hasta primaria completa & 4,94 & 1,29 & 0,95 & 0,59 & 13,88 & 1,37 & 1,32 & 19,40 & 24,34 \\
\hline Estudios secundarios & 6,79 & 2,31 & 3,77 & 1,36 & 14,04 & 4,59 & 3,59 & 29,66 & 36,45 \\
\hline Estudios superiores & 14,14 & 4,28 & 5,23 & 7,36 & 17,38 & 3,67 & 4,80 & 42,71 & 56,86 \\
\hline \multicolumn{10}{|l|}{ CLASE SOCIAL (EGP) } \\
\hline $\begin{array}{l}\text { Clase de servicios } \\
\text { (profesional / gerencial) }\end{array}$ & 13,33 & 5,62 & 5,97 & 8,31 & 22,23 & 5,83 & 4,19 & 52,15 & 65,48 \\
\hline $\begin{array}{l}\text { Empleados no } \\
\text { manuales rutinarios }\end{array}$ & 10,40 & 3,24 & 6,20 & 5,31 & 20,75 & 2,14 & 4,69 & 42,32 & 52,72 \\
\hline $\begin{array}{l}\text { Pequeña burguesía } \\
\text { (pequeño empleador) }\end{array}$ & 7,76 & 3,83 & 1,68 & 1,28 & 12,71 & 3,25 & 3,40 & 26,15 & 33,91 \\
\hline $\begin{array}{l}\text { Trabajadores manuales } \\
\text { calificados }\end{array}$ & 8,25 & 0,79 & 1,42 & 0,15 & 12,31 & 5,38 & 2,06 & 22,10 & 30,35 \\
\hline $\begin{array}{l}\text { Trabajadores manuales } \\
\text { no calificados }\end{array}$ & 2,66 & 1,15 & 1,42 & 0,41 & 9,91 & 1,01 & 2,34 & 16,23 & 18,89 \\
\hline $\begin{array}{l}\text { Sin ocupación / } \\
\text { no especificada }\end{array}$ & 6,97 & 1,69 & 2,31 & 2,12 & 10,98 & 2,45 & 1,16 & 20,71 & 27,69 \\
\hline \multicolumn{10}{|l|}{$\begin{array}{l}\text { QUINTIL INGRESO } \\
\text { PER CAPITA (3 grupos) }\end{array}$} \\
\hline $1+2$ & 2,46 & 1,68 & 1,22 & 0,34 & 13,35 & 1,08 & 1,66 & 19,32 & 21,78 \\
\hline $3+4$ & 6,94 & 3,32 & 3,41 & 0,80 & 17,29 & 6,05 & 5,24 & 36,10 & 43,04 \\
\hline 5 & 22,97 & 2,86 & 5,27 & 6,78 & 18,02 & 3,60 & 4,78 & 41,32 & 64,29 \\
\hline Sin especificar & 9,07 & 2,06 & 4,92 & 7,15 & 10,67 & 2,20 & 0,74 & 27,74 & 36,81 \\
\hline \multicolumn{10}{|c|}{ TAMAÑO DE LA LOCALIDAD } \\
\hline Grande & 7,98 & 2,89 & 4,22 & 4,69 & 15,55 & 5,13 & 3,54 & 36,02 & 43,99 \\
\hline Mediana & 9,56 & 3,18 & 2,91 & 1,07 & 12,65 & 3,50 & 3,17 & 26,47 & 36,03 \\
\hline Pequeña / Rural & 7,70 & 1,84 & 2,01 & 0,57 & 14,39 & 1,13 & 2,60 & 22,53 & 30,23 \\
\hline \multicolumn{10}{|l|}{ AFILIACIÓN } \\
\hline Sí & - & 2,64 & 3,72 & 3,78 & 18,79 & 2,42 & 3,07 & 34,42 & 47,15 \\
\hline No & - & 2,11 & 2,15 & 0,58 & 8,15 & 4,58 & 3,14 & 20,70 & 20,70 \\
\hline
\end{tabular}

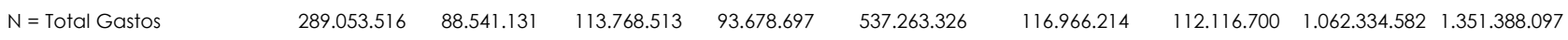

Fuente: Elaboración propia en base a Encuesta Nacional 2005, Centro de Estudios de Opinión Pública, Universidad de Buenos Aires (CEDOP-UBA) y Dirección de Estadísticas e Información en Salud, Ministerio de Salud (DEIS-MSN). 
gasto en análisis, tratamientos e internaciones, las consultas al dentista y los otros gastos, con más de un $8 \%$ cada uno. La menor participación corresponde al gasto en consulta médica $(6,5 \%)$.

Al pasar de 2003 a 2005 el promedio de gasto total en salud habría crecido un 30\% (Cuadro 3a). Salvo la consulta al dentista, todos los promedios de gasto crecen al pasar de 2003 a 2005. Sin dudas, el estudio comparativo del gasto en salud con mediciones anteriores se ve afectado por las variaciones en el valor de la moneda. Sin embargo, como información contextual puede señalarse que el valor del galeno creció menos de un $8 \%$ (pasó de $\$ 6,50$ a $\$ 7,00$ pesos) y el dólar se mantuvo estable (alrededor de $\$ 2,90$ pesos). A su vez, el índice de precios al consumidor para "Atención médica y gastos para la salud" (que incluye sólo Afiliación voluntaria y Medicamentos, lo que más varía) creció un $12 \%$ de 2003 a 2005. Para evitar tratamientos dudosos de los datos, y basados en lo acotado de las variaciones de los indicadores anteriores, se prefiere una comparación a valores nominales. De todas formas, lo que se compara fundamentalmente son las participaciones porcentuales en el gasto total, además de las relaciones del gasto del quintil más alto de ingresos respecto de los más bajos.
De 2003 a 2005 el gasto en medicamentos disminuye su participación en el gasto total en salud (disminuye algo más de un $6 \%$, que implica una baja de un $14 \%$ de su peso en 2003), manteniendo su participación dentro del gasto de los afiliados (sin considerar lo gastado de bolsillo en afiliación voluntaria) y perdiendo participación en el gasto de los no afiliados (los datos con distinción de gastos de afiliados no se presentan aquí). Por su lado, la consulta médica se ha mantenido en general, pero perdiendo algo de terreno en el gasto de los afiliados y ganando fuertemente terreno entre los no afiliados.

Se señaló que el promedio de gasto global en salud habría aumentado un 30\%. Ello no sería uniforme para todos los sectores, ya que mientras el promedio de gastos del quintil más alto de ingresos prácticamente se habría mantenido, el de los sectores medios de ingresos habría crecido un $26 \%$ y el de los bajos (quintiles $1+2$ ) un $46 \%$. O sea, el crecimiento del total del gasto en salud sería producto del crecimiento en los sectores de ingresos bajos y medios. En el bienio 2003-2005, crecería el gasto en salud del sector más pobre, seguido por los sectores medios. El rubro medicamentos es el que menos crece en el sector pobre (fuera de la caída del promedio de gasto en consultas al dentista en todos los sectores

Cuadro 3a. COCIENTES DEL GASTO EN SALUD DEL QUINTIL DE MAYORES INGRESOS SOBRE LOS DE MENORES INGRESOS EN 2003 Y EN 2005, Y COCIENTES DE LOS QUINTILES DE 2005 EN LOS RESPECTIVOS DE 2003, INCLUYENDO COCIENTES DE TOTALES DE 2005 EN 2003. POBLACIÓN DE O AÑOS Y MÁS. ARGENTINA.

\begin{tabular}{|c|c|c|c|c|c|c|}
\hline \multirow[b]{2}{*}{ TIPO DE GASTO } & \multicolumn{6}{|c|}{ COCIENTES } \\
\hline & $\begin{array}{l}\text { AÑO } 2005 \\
5^{\circ} / 1^{\circ}+2^{\circ}\end{array}$ & $\begin{array}{l}\text { AÑO } 2003 \\
5^{\circ} / 1^{\circ}+2^{\circ}\end{array}$ & $\begin{array}{c}5^{\circ} 2005 / / \\
5^{\circ} 2003\end{array}$ & $\begin{array}{c}2^{\circ}+3^{\circ} 2005 / \\
2^{\circ}+3^{\circ} 2003\end{array}$ & $\begin{array}{c}1^{\circ} 2005 / / \\
1^{\circ} 2003\end{array}$ & $\begin{array}{c}\text { TOTAL } 2005 \text { / } \\
\text { TOTAL } 2003\end{array}$ \\
\hline Afiliación voluntaria & 9,34 & 29,16 & 1,18 & 1,46 & 3,67 & 1,60 \\
\hline Consultas al médico & 1,70 & 5,79 & 0,62 & 1,41 & 2,10 & 1,32 \\
\hline Consultas al dentista & 4,32 & 4,57 & 0,68 & 0,86 & 0,72 & 0,93 \\
\hline Consultas otros profesionales & 19,94 & 26,00 & 1,24 & 1,11 & 1,62 & 1,95 \\
\hline Medicamentos & 1,35 & 1,57 & 1,16 & 1,05 & 1,34 & 1,12 \\
\hline Análisis, Tratamientos, Internaciones & 3,33 & 6,63 & 1,04 & 2,00 & 2,08 & 1,84 \\
\hline Otros Gastos & 2,88 & 4,52 & 0,99 & 1,85 & 1,55 & 1,40 \\
\hline Total & 2,95 & 4,11 & 1,05 & 1,26 & 1,46 & 1,30 \\
\hline
\end{tabular}


de ingreso). Los que más crecen son la afiliación voluntaria y la consulta médica en este sector. Los gastos en consultas del sector de altos ingresos, en particular la consulta médica, son los únicos que caen en el bienio considerado, dentro de un contexto de estabilidad global del gasto en salud de ese sector al pasar de 2003 a 2005. Un avance particular corresponde tanto al crecimiento de la afiliación en sí como del gasto en afiliación dentro de los sectores de menores ingresos (particularmente la afiliación a obras sociales en las localidades pequeñas y zonas rurales). Le sigue, siempre dentro del sector de bajos ingresos, el crecimiento del gasto promedio en consulta médica (se duplica). En realidad, se recupera de los niveles bajos de 2003.

Una primera observación somera sobre desigualdad en salud es ver las diferencias entre el promedio de gasto en salud del quintil superior de ingresos $\left(5^{\circ}\right)$ y el del inferior $\left(1^{\circ}\right)$, tanto en 2005 como en 2003. Debe aclararse que en este ejercicio queda fuera un $17 \%$ de la población, ya que este porcentaje rehusó informar sobre los ingresos de su hogar. A efectos comparativos con 2003 y teniendo en cuenta el tamaño muestral, se consideran tres grupos: $1^{\circ}$ y $2^{\circ}$ quintil sumados, luego $3^{\circ}$ y $4^{\circ}$ y finalmente el $5^{\circ}$ quintil.

Comenzando por el gasto de la población en general, el promedio del segmento de mayores ingresos ( $5^{\circ}$ quintil) triplica al de menores ingresos (suma de $1^{\circ}$ y $2^{\circ}$ quintil). La diferencia mayor corresponde a la consulta con otros profesionales (aunque con pocos casos) seguida por la afiliación voluntaria (el promedio de gastos del sector alto es 9 veces el del sector bajo) y la consulta con el dentista (4 veces). Los que menos varían son el promedio de gastos en medicamentos (el gasto promedio del sector de altos ingresos es un 1,35 veces el del bajo) y el de consulta médica (1,7 veces).

Al pasar de 2003 a 2005 el cociente del promedio de gasto global en salud del sector de altos ingresos respecto de los bajos cae de 4 veces a 3 veces. Y esto es particularmente notorio por la caída de las diferencias en afiliación voluntaria (el cociente pasa de 29 veces a 9 veces) y de las de consulta médica (de cerca de 6 veces cae a menos de 2 veces). En el sector de menores ingresos lo que crece es la participación del gasto en afiliación voluntaria, la de la consulta médica y la del rubro de análisis, tratamientos e internaciones. Cae la participación de los gastos en consulta al dentista y en medicamentos.

Estas comparaciones llevan a sugerir que la desigualdad en el gasto en salud (gruesamente medida) habría disminuido en 2005. Lo que sería resultado, fundamentalmente, del mayor gasto del sector de bajos ingresos -particularmente la mayor participación que adquieren entre ellos la protección y la consulta médica (Cuadro 3b)-, atento a la estabilidad en el bienio 2003-2005 del promedio de gasto en los distintos rubros del quintil superior de ingresos, incluyendo la caída del gasto en consultas (al médico y al dentista) de este sector. Y es interesante señalar que ello ocurre en rubros distintos a medicamentos, cuya participación porcentual es casi la única que cae en el gasto total del sector pobre, dejando de lado la caída del promedio de gasto en consultas al dentista en todos los niveles de ingresos (Cuadro 3b).

\subsection{CONDICIONES DE SALUD Y DIFERENCIALES SOCIOECONÓMICOS EN SALUD: UNA COMPARA- CIÓN INTERNACIONAL}

No es novedad la referencia a una asociación entre la posición socioeconómica de los individuos y su estado de salud. Otra cuestión es la posibilidad de especificación de esta relación. Para el caso de Japón, Ishida señala la ausencia de estudios que relacionen ambas variables en su país, a la par que desarrolla un trabajo sobre el tema aprovechando una investigación sobre salud que contemplaba variables de estratificación social. El autor argumenta que esta falta de estudios podría deberse al supuesto de la ausencia de desigualdad en salud en su país.

Hay una distancia, sin dudas, entre la situación descripta para Japón y Argentina. No hay un seguro universal de salud a nivel local, aunque debe señalarse que, dentro de parámetros comparativos internacionales, el funcionamiento del sistema público de salud local cumple un razonable rol supletorio. La posibilidad de comparar resultados locales tomando como parámetro Japón resulta entonces de indudable interés para evaluar los diferenciales socioeconómicos en salud. 


\section{Cuadro 3b. PESO PORCENTUAL DE CADA GASTO EN EL TOTAL DEL GASTO DE QUINTILES DE INGRESO PER} CAPITA AGRUPADOS.

\begin{tabular}{|c|c|c|c|c|c|c|c|c|}
\hline \multirow[b]{2}{*}{ TIPO DE GASTO } & \multicolumn{4}{|c|}{ AÑO 2005} & \multicolumn{4}{|c|}{ AÑO 2003} \\
\hline & $1^{\circ}-2^{\circ}$ QUINTIL & $3^{\circ}-4^{\circ}$ QUINTIL & $5^{\circ}$ QUINTIL & TOTAL & $1^{\circ}-2^{\circ}$ QUINTIL & $3^{\circ}-4^{\circ}$ QUINTIL & $5^{\circ}$ QUINTIL & TOTAL \\
\hline Afiliación voluntaria & $11,3 \%$ & $16,1 \%$ & $35,7 \%$ & $21,4 \%$ & $4,5 \%$ & $13,9 \%$ & $31,9 \%$ & $17,4 \%$ \\
\hline Consultas al médico & $7,7 \%$ & $7,7 \%$ & $4,4 \%$ & $6,5 \%$ & $5,4 \%$ & $6,9 \%$ & $7,6 \%$ & $6,4 \%$ \\
\hline Consultas al dentista & $5,6 \%$ & $7,9 \%$ & $8,2 \%$ & $8,4 \%$ & $11,4 \%$ & $11,7 \%$ & $12,7 \%$ & $11,7 \%$ \\
\hline Consultas otros profesionales & $1,6 \%$ & $1,9 \%$ & $10,5 \%$ & $6,9 \%$ & $1,4 \%$ & $2,1 \%$ & $8,9 \%$ & $4,6 \%$ \\
\hline Medicamentos & $61,3 \%$ & $40,2 \%$ & $28,0 \%$ & $39,8 \%$ & $66,7 \%$ & $48,3 \%$ & $25,5 \%$ & $46,0 \%$ \\
\hline Análisis, Tratamientos, Internaciones & $5,0 \%$ & $14,1 \%$ & $5,6 \%$ & $8,7 \%$ & $3,5 \%$ & $8,9 \%$ & $5,6 \%$ & $6,1 \%$ \\
\hline Otros Gastos & $7,6 \%$ & $12,2 \%$ & $7,4 \%$ & $8,3 \%$ & $7,2 \%$ & $8,3 \%$ & $7,9 \%$ & $7,7 \%$ \\
\hline Total & $100,0 \%$ & $100,0 \%$ & $100,0 \%$ & $100,0 \%$ & $100,0 \%$ & $100,0 \%$ & $100,0 \%$ & $100,0 \%$ \\
\hline
\end{tabular}

Fuente: Elaboración propia en base a Encuesta Nacional 2003 y 2005, Centro de Estudios de Opinión Pública, Universidad de Buenos Aires (CEDOP-UBA) y Dirección de Estadísticas e Información en Salud, Ministerio de Salud de la Nación (DEIS-MSN).

Dentro de este enfoque comparativo, evaluaremos cuatro hipótesis consideradas por Ishida, quien en su selección sigue investigaciones de otros países. La hipótesis 1 propone que "los trabajadores no manuales, particularmente los profesionales y gerentes, tienen mejores condiciones de salud que los trabajadores manuales, porque sus condiciones de trabajo son relativamente más favorables que aquellas de la clase obrera manual". La hipótesis 2 propone que "los logros educacionales incrementan la posibilidad de mantener buena salud". La hipótesis 3 afirma que se espera que "los niveles de ingreso afecten resultados relacionados a salud". Finalmente, la hipótesis 4 señala que "la tenencia de activos afecta las condiciones de salud". Esta última hipótesis es evaluada con una alternativa de autopercepción de posición social, porque en el relevamiento local no se indagó sobre tenencia de bienes en el hogar.

En ambos países (personas de 20 años y más), algo más de un $40 \%$ señala la presencia de al menos una enfermedad crónica y algo más del $70 \%$ visitó al médico (o al dentista en Japón) en el último año. Un 10\% en Japón, el doble en Argentina, señala que tuvo alguna restricción en su trabajo o tareas del hogar debido a la presencia de enfermedades o dolencias. En cuanto a la percepción de la propia salud como no buena o mala, un $15 \%$ en Japón (pobre o no buena) y un
$19 \%$ en la Argentina (de regular a muy mala) menciona ese tipo de condición.

Con el objeto de mostrar que si bien las variables dependientes están interrelacionadas pero que miden condiciones diferentes, Ishida da cuenta de que las correlaciones entre ellas "no son excepcionalmente altas". Los resultados en ambos países favorecen la consideración de cada una de estas condiciones.

En el Cuadro 4, referido a las condiciones de salud crónicas, se muestran resultados comparativos para tres modelos. El Modelo 1, dada la importancia atribuida a la clase social, considera la misma usando como control el sexo y la edad. Ishida señala que entre la relación bivariada de clase y la presencia de enfermedades crónicas hay una asociación significativa alta, lo que no se da a nivel local. Cuando se controla por sexo y edad, tal relación desaparece en Japón. Para ambas ecuaciones, la edad es altamente significativa, pero, además, en Argentina el trabajo rural se vincula significativamente a las enfermedades crónicas (cuando se controla por sexo y edad). Ishida mismo señala que el trabajo rural se vincula a las enfermedades crónicas, pero que al introducir la variable edad -los trabajadores rurales tienden a ser mayores-, esa relación desaparece en su caso. Por el contrario, es la presencia del sexo y la edad junto a la clase social lo que hace aparecer tal vinculación en Argentina. 
En el Modelo 2 se incorpora tanto los años de educación como el tamaño de las ciudades. Prácticamente los resultados de ambas ecuaciones son similares, con la única presencia significativa de la edad, dentro de pautas esperadas. En el caso japonés, aunque de forma limitada, en las ciudades medianas es menos probable la presencia de enfermedades crónicas que en las pequeñas y rurales. Tal tendencia no se da en los datos locales.

El Modelo 3 incorpora, para Japón, ingreso y presencia de activos en el hogar, ingreso y auto-percepción de posición social en
Argentina. Las pautas son más o menos similares para ambos países. El efecto de la edad es dominante y no habría diferenciales socioeconómicos para la presencia de enfermedades crónicas.

En el Cuadro 5 se presentan ecuaciones buscando predecir visitas al médico. Nótese que Ishida usa esta variable como un "proxy" de disponibilidad o accesibilidad a cuidados de salud. Los tres primeros modelos repiten las variables independientes consideradas anteriormente, mientras que el Modelo 4 agrega enfermedades crónicas. En todos los modelos se observa la presencia determinante de la edad para concurrir al

CUadro 4. REGRESIONES LOGÍSTICAS PARA PREDECIR CONDICIONES DE SALUD CRÓNICAS. POBLACIÓN DE 20 AÑOS Y MÁS. ARGENTINA 2005, JAPÓN 2000.

\begin{tabular}{|c|c|c|c|c|c|c|}
\hline & \multicolumn{2}{|c|}{ MODELO I } & \multicolumn{2}{|c|}{ MODELO 2} & \multicolumn{2}{|c|}{ MODELO 3} \\
\hline & JAPÓN & ARGENTINA & JAPÓN & ARGENTINA & JAPÓN & ARGENTINA \\
\hline \multicolumn{7}{|l|}{ VARIABLES INDEPENDIENTES } \\
\hline Varones & $-0,142$ & $-0,222$ & $-0,140$ & $-0,222$ & $-0,153$ & $-0,261$ \\
\hline Edad & $0,061 * *$ & $0,055^{* *}$ & $0,061^{* *}$ & $0,055^{* *}$ & $0,061^{* *}$ & $0,055^{* *}$ \\
\hline Clase de servicios (profesional / gerencial) & - & - & - & - & - & - \\
\hline Empleados no manuales rutinarios & $-0,141$ & 0,065 & $-0,167$ & 0,043 & $-0,161$ & $-0,053$ \\
\hline Pequeña burguesía (pequeño empleador) & 0,253 & $-0,395$ & 0,219 & $-0,420$ & 0,220 & $-0,493$ \\
\hline Trabajo rural & $-0,286$ & $0,793 *$ & $-0,410$ & 0,642 & $-0,407$ & 0,603 \\
\hline Trabajadores manuales calificados & 0,225 & 0,150 & 0,156 & 0,110 & 0,181 & $-0,015$ \\
\hline Trabajadores manuales no calificados & $-0,210$ & 0,000 & $-0,232$ & $-0,056$ & $-0,225$ & $-0,138$ \\
\hline Educación (en años) & - & - & $-0,010$ & $-0,005$ & $-0,009$ & $-0,006$ \\
\hline \multicolumn{7}{|l|}{ Tamaño de la localidad } \\
\hline Grande & - & - & $-0,211$ & $-0,187$ & $-0,218$ & $-0,072$ \\
\hline Mediana & - & - & $-0,277+$ & $-0,206$ & $-0,282+$ & $-0,194$ \\
\hline Ingreso & - & - & - & - & 0,002 & 0,000 \\
\hline Ingreso no declarado & - & - & - & - & $-0,027$ & $-0,555^{*}$ \\
\hline Activos hogar / Posición social & - & - & - & - & $-0,039$ & $-0,045$ \\
\hline Constante & $-3,524 * *$ & $-2,616 * *$ & $-3,424 * *$ & $-2,406 * *$ & $-3,332 * *$ & $-2,103 * *$ \\
\hline-2 log de la verosimilitud & $1.407,334$ & $1.051,451$ & $1.398,209$ & $1.049,835$ & $1.396,337$ & $1.037,578$ \\
\hline Número de casos & 1.193 & 865 & 1.186 & 865 & 1.186 & 865 \\
\hline
\end{tabular}

Nota: ${ }^{* *} \mathrm{p}<0,01 ; * \mathrm{p}<0,05 ;+\mathrm{p}<0,10$

Fuente: Elaboración propia en base a datos para Argentina de la Encuesta Nacional 2005, Centro de Estudios de Opinión Pública, Universidad de Buenos Aires (CEDOP-UBA) y Dirección de Estadísticas e Información en Salud, Ministerio de Salud de la Nación (DEIS-MSN); y datos para Japón de la Encuesta Ómnibus Nacional 2000, Central Research Services, Módulo Salud Agregado. 
CUadro 5. REGRESIONES LOGÍSTICAS PARA PREDECIR CONSULTAS AL MÉDICO. POBLACIÓN DE 20 AÑOS Y MÁS. ARGENTINA 2005, JAPÓN 2000.

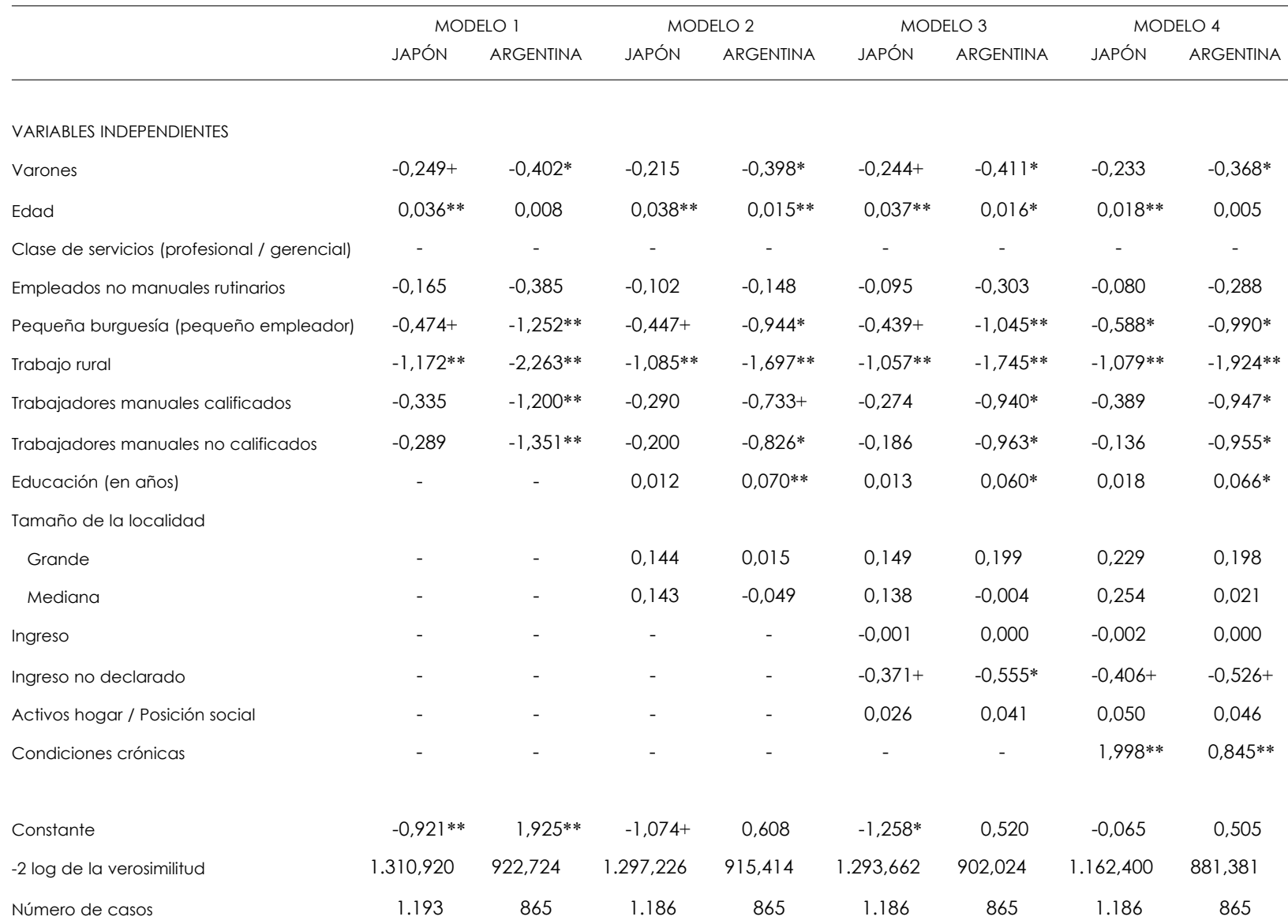

Nota: $* * \mathrm{p}<0,01 ; * \mathrm{p}<0,05 ;+\mathrm{p}<0,10$

Fuente: Elaboración propia en base a datos para Argentina de la Encuesta Nacional 2005, Centro de Estudios de Opinión Pública, Universidad de Buenos Aires (CEDOP-UBA) y Dirección de Estadísticas e Información en Salud, Ministerio de Salud de la Nación (DEIS-MSN); y datos para Japón de la Encuesta Ómnibus Nacional 2000, Central Research Services, Módulo Salud Agregado.

médico en Japón, mientras que para Argentina ello no se observa en los Modelos 1 ni 4 . Para el caso local, casi todas las variables de clase en el Modelo 1 son negativas y significativas, a excepción de los trabajadores no manuales rutinarios. La pequeña burguesía y los trabajadores manuales tendrían menos chances de visitar al médico que aquellos que ocupan la cúspide de la pirámide (la clase de servicios). En el caso japonés, son la pequeña burguesía y los trabajadores rurales los que exhiben menos posibilidades de concurrir al médico comparados con la clase de servicios (f). Estos efectos persisten, como nota Ishida, cuando se controla por años de educación, tamaño de ciudad e ingresos. La diferencia es, para el caso local, que cuando se introducen estos controles la edad también es significativa en Argentina, agregándose que los años de educación tienen una presencia positiva significativa en Argentina, mostrando, en estos casos, las mayores chances de quienes tienen altos niveles de educación de concurrir al médico. Por el contrario, la educación no se asocia en Japón a la visita al médico.

Entonces, a la persistencia del efecto de la edad y la clase en Japón, en Argentina se agrega también, además de la persistencia de la clase, 
la vinculación positiva significativa de la visita al médico dada por la edad y la educación. O sea, en el análisis de las visitas al médico están presentes diferenciales socioeconómicos en ambos países, más allá de algunos matices. La diferencia local que puede señalarse es la baja chance de concurrir al médico de los trabajadores manuales comparados con la clase profesional-gerencial, lo que no se da en Japón. En Japón, sociedad particularmente envejecida, la edad es significativa en todos los modelos, mientras que en Argentina es significativa cuando se toma en cuenta además la educación y el ingreso, lo que desaparece cuando se introducen las condiciones crónicas (que se supone fuertemente vinculada a la edad). Es particularmente más notoria en Argentina que en Japón la mayor chance de visitar al médico de las mujeres, comparando con la de los varones.

Cuando se considera la restricción de actividades en el trabajo debido a condiciones físicas (Cuadro 6), los cuatro primeros modelos repiten las variables independientes consideradas para la visita al médico, mientras que el Modelo 5 agrega estas visitas al médico como una variable

CUadro 6. REGRESIONES LOGÍSTICAS PARA PREDECIR RESTRICCIÓN DE ACTIVIDADES. POBLACIÓN DE 20 AÑOS Y MÁS. ARGENTINA 2005, JAPÓN 2000.

\begin{tabular}{|c|c|c|c|c|c|c|c|c|c|c|}
\hline & \multicolumn{2}{|c|}{ MODELO 1} & \multicolumn{2}{|c|}{ MODELO 2} & \multicolumn{2}{|c|}{ MODELO 3} & \multicolumn{2}{|c|}{ MODELO 4} & \multicolumn{2}{|c|}{ MODELO 5} \\
\hline & JAPÓN & ARGENTINA & JAPÓN & ARGENTINA & JAPÓN & ARGENTINA & JAPÓN & ARGENTINA & JAPÓN & ARGENTINA \\
\hline \multicolumn{11}{|l|}{ VARIABLES INDEPENDIENTES } \\
\hline Varones & $-0,220$ & $-0,143$ & $-0,214$ & $-0,147$ & $-0,188$ & $-0,155$ & $-0,176$ & $-0,100$ & $-0,168$ & $-0,082$ \\
\hline Edad & $0,043^{*} *$ & $0,025^{* *}$ & $0,035^{* *}$ & $0,020 * *$ & $0,033^{* *}$ & $0,020 * *$ & $0,016+$ & 0,010 & 0,013 & 0,008 \\
\hline Clase de servicios (profesional / gerencial) & - & - & - & - & - & - & - & - & - & - \\
\hline Empleados no manuales rutinarios & 0,371 & $-0,382$ & 0,175 & $-0,578+$ & 0,184 & $-0,644 *$ & 0,245 & $-0,647^{*}$ & 0,246 & $-0,641 *$ \\
\hline Pequeña burguesía (pequeño empleador) & $0,604+$ & $-0,246$ & 0,334 & $-0,504$ & 0,351 & $-0,564+$ & 0,281 & $-0,493$ & 0,366 & $-0,405$ \\
\hline Trabajo rural & 0,041 & $0,721+$ & $-0,371$ & 0,376 & $-0,449$ & 0,285 & $-0,347$ & 0,171 & $-0,234$ & 0,475 \\
\hline Trabajadores manuales calificados & $0,871^{* *}$ & 0,047 & 0,480 & $-0,339$ & 0,418 & $-0,432$ & 0,401 & $-0,437$ & 0,468 & $-0,356$ \\
\hline Trabajadores manuales no calificados & 0,364 & $-0,241$ & 0,038 & $-0,665+$ & $-0,042$ & $-0,756^{*}$ & 0,044 & $-0,748+$ & 0,035 & $-0,688+$ \\
\hline Educación (en años) & - & - & $-0,127 *$ & $-0,054 *$ & $-0,102$ & $-0,047+$ & $-0,097$ & $-0,046+$ & $-0,094$ & $-0,057^{*}$ \\
\hline \multicolumn{11}{|l|}{ Tamaño de la localidad } \\
\hline Grande & - & - & $-0,284$ & 0,204 & $-0,336$ & 0,269 & $-0,302$ & 0,282 & $-0,310$ & 0,232 \\
\hline Mediana & - & - & $-0,124$ & 0,202 & $-0,107$ & 0,207 & $-0,028$ & 0,249 & $-0,051$ & 0,227 \\
\hline Ingreso & - & - & - & - & $-0,002$ & 0,000 & $-0,003$ & 0,000 & $-0,003$ & 0,000 \\
\hline Ingreso no declarado & - & - & - & - & $-0,142$ & $-0,448+$ & $-0,142$ & $-0,341$ & $-0,106$ & $-0,273$ \\
\hline Activos hogar / Posición social & - & - & - & - & $-0,114+$ & $-0,041$ & $-0,114+$ & $-0,035$ & $-0,126+$ & $-0,045$ \\
\hline Condiciones crónicas & - & - & - & - & - & - & $1,384^{* *}$ & $0,902 * *$ & $1,108 * *$ & $0,803^{* *}$ \\
\hline Consultas al médico & - & - & - & - & - & - & - & - & $1,301 * *$ & $0,929 * *$ \\
\hline Constante & $-4,520 * *$ & $-2,159 * *$ & $-2,713^{* *}$ & $-1,241 *$ & $-2,236 * *$ & $-0,933$ & $-1,468$ & $-1,000$ & $-1,705+$ & $-1,494^{*}$ \\
\hline-2 log de la verosimilitud & 755,448 & 901,426 & 741,209 & 895,955 & 735,546 & 892,250 & 698,058 & 867,579 & 685,230 & 850,370 \\
\hline Número de casos & 1.193 & 865 & 1.186 & 865 & 1.186 & 865 & 1.186 & 865 & 1.186 & 865 \\
\hline
\end{tabular}

Nota: $* * \mathrm{p}<0,01 ; * \mathrm{p}<0,05 ;+\mathrm{p}<0,10$

Fuente: Elaboración propia en base a datos para Argentina de la Encuesta Nacional 2005, Centro de Estudios de Opinión Pública, Universidad de Buenos Aires (CEDOP-UBA) y Dirección de Estadísticas e Información en Salud, Ministerio de Salud de la Nación (DEIS-MSN); y datos para Japón de la Encuesta Ómnibus Nacional 2000, Central Research Services, Módulo Salud Agregado. 
adicional, con la idea de controlar por acceso o disponibilidad. En los tres primeros modelos, antes de la entrada de variables de condiciones de salud como variables independientes, la edad es significativamente positiva en todos estos casos, indicando que la restricción de actividades se asocia claramente con el aumento de la edad. Como nota Ishida, en realidad este efecto de la edad prácticamente desaparece cuando se controla por la presencia de enfermedades crónicas y visitas al médico, ya que estas últimas se vinculan positivamente con la edad.

En el Modelo 1, en el caso japonés la pequeña burguesía y el trabajo manual calificado se vinculan a la restricción, mientras que en Argentina es el trabajo rural el que muestra alguna tendencia en este sentido. Estos sectores mostrarían mayores restricciones comparados con la clase profesional-gerencial en ambas muestras, controlando por sexo y edad. Cuando en el Modelo 2 se introducen los años de educación y el tamaño de las localidades, en ambos casos además de la edad la baja educación se vincula a estas restricciones. En el caso argentino, la presencia de trabajadores no manuales rutinarios no se vincularía a estas restricciones, sorprendiendo que esa misma ligera tendencia se dé para los trabajadores manuales no calificados. Y ello se mantiene para los distintos modelos. Al controlar en Japón la educación y/o los activos del hogar (auto-percepción de posición social en nuestro caso), desaparece la relevancia de la pequeña burguesía y de los trabajadores manuales calificados. En cambio, en Argentina surgen las presencias señaladas.

En el Modelo 3 (con presencia de ingresos y activos del hogar, o percepción de posición social) disminuye la significación de la baja educación en Argentina y desaparece en Japón. Los trabajadores no manuales rutinarios muestran ya una mayor significación en su relación negativa con restricciones, a la par del mantenimiento de la ligera significación de los trabajadores manuales no calificados en su vinculación negativa con restricción de actividades. En el Modelo 4, la edad todavía es ligeramente significativa en Japón y desaparece esa significación en Argentina. En ambas muestras, la presencia de enfermedades crónicas es altamente significativa, a costa de la variable edad. Sigue aquí a nivel local la ligera relevancia de la baja educación (los encuestados con mayor educación tenderían a vincularse menos con las restricciones). Es probable que ello explique, en alguna medida, la cierta relevancia negativa de los extremos de clase en Argentina (dejando de lado la clase en la cúspide -profesional, gerencial-), lo que no ocurre en Japón. Como se señalara, que las restricciones físicas se asocien negativamente con las clases medias no manuales estaría dentro de pautas esperadas, no así la ligera vinculación del mismo signo de la clase más baja.

En el Modelo 5, fuera de las diferencias para educación, trabajadores no manuales rutinarios y manuales no calificados, en ambos casos es fuerte la presencia de enfermedades crónicas y visitas al médico para aumentar las restricciones físicas en el trabajo o las tareas del hogar. En el caso japonés los encuestados con enfermedades crónicas exhibían cerca de tres veces más chances de tener restricción de actividades comparados con aquellos que no tenían enfermedades crónicas, mientras que este valor trepaba a 3,7 veces para las chances de tener restricción de actividades por parte de los que visitaron al médico en el último año, comparados con los que no lo hicieron. Los valores locales fueron 3,0 y 2,3 veces respectivamente (g). Resulta más importante a nivel local la presencia de enfermedades crónicas, las visitas al médico en Japón.

En una síntesis para Japón, el autor señala que fuera de las condiciones de salud (enfermedades crónicas y visitas al médico), el único factor socioeconómico que afecta las chances de tener restricciones es la presencia de activos en el hogar. Para Argentina, el factor relevante -fuera de las condiciones de salud- es la baja educación y la presencia negativa de las distintas clases comparadas con la clase profesional-gerencial (aunque solo es significativa la presencia negativa de los trabajadores no manuales rutinarios). En general, con leves diferencias, podría puntualizarse que lo central para dar cuenta de las restricciones a las actividades son las condiciones de salud en ambos países, con el agregado de que en Japón la tenencia de activos favorece la no presencia de tales restricciones, mientras que en Argentina ese efecto correspondería a los niveles altos de educación y a la clase en la cúspide.

Finalmente, la última variable considerada es la propia percepción de su estado de salud por parte de los encuestados, analizándose 
los factores asociados a una percepción desfavorable de la propia salud. En el caso japonés los hombres exhiben 1,4 veces más chances de informar un estado de salud razonable o bueno que las mujeres, siendo esta tendencia consistente a lo largo de los distintos modelos (8 p.17). En el caso argentino (ver Cuadro 7) esta variable es no significativa, indicando ausencia de diferencias por sexo. En ambos casos, con el avance de la edad se tiende a informar un nivel bajo de salud. En la muestra japonesa, esta tendencia se mantiene aun ante la presencia de enfermedades crónicas, mientras que en la muestra argentina desaparece el efecto edad en ese caso.
En lo que se refiere a la clase social, sus efectos son importantes en ambas situaciones. En Japón, la pequeña burguesía y los trabajadores manuales tienen mayores chances de informar un estado de salud desfavorable comparados con la clase de servicios (profesionales y gerentes). En Argentina, todas las clases remanentes consideradas tienen más chances de dar cuenta de un estado de salud desfavorable, comparados con la clase de servicios. $Y$ estas tendencias se mantienen, en ambos casos, cuando se controlan condiciones de salud como enfermedades crónicas o visitas al médico (tomada esta última como accesibilidad a condiciones de salud, según Ishida).

CUadro 7. REGRESIONES LOGÍSTICAS PARA PREDECIR AUTO-PERCEPCIÓN DE SALUD. POBLACIÓN DE 20 AÑOS Y MÁS. ARGENTINA 2005, JAPÓN 2000.

\begin{tabular}{|c|c|c|c|c|c|c|c|c|c|c|}
\hline & \multicolumn{2}{|c|}{ MODELO 1} & \multicolumn{2}{|c|}{ MODELO 2} & \multicolumn{2}{|c|}{ MODELO 3} & \multicolumn{2}{|c|}{ MODELO 4} & \multicolumn{2}{|c|}{ MODELO 5} \\
\hline & JAPÓN & ARGENTINA & JAPÓN & ARGENTINA & JAPÓN & ARGENTINA & JAPÓN & ARGENTINA & JAPÓN & ARGENTINA \\
\hline \multicolumn{11}{|l|}{ VARIABLES INDEPENDIENTES } \\
\hline Varones & $-0,312+$ & 0,060 & $-0,350+$ & 0,053 & $-0,343+$ & 0,065 & $-0,367+$ & 0,291 & $-0,356+$ & 0,287 \\
\hline Edad & $0,038 * *$ & $0,037 * *$ & $0,042 * *$ & $0,021 * *$ & $0,040 * *$ & $0,022 * *$ & $0,016^{*}$ & $-0,002$ & $0,014+$ & $-0,003$ \\
\hline Clase de servicios (profesional / gerencial) & - & - & - & - & - & - & - & - & - & - \\
\hline Empleados no manuales rutinarios & $-0,074$ & $1,807 * *$ & $-0,048$ & $1,144+$ & $-0,036$ & $1,148+$ & 0,036 & $1,233+$ & 0,047 & $1,225+$ \\
\hline Pequeña burguesía (pequeño empleador) & $0,633^{*}$ & $2,068 * *$ & $0,719 *$ & $1,221+$ & $0,760 *$ & $1,193+$ & $0,780 *$ & $1,291+$ & $0,882 *$ & $1,311 *$ \\
\hline Trabajo rural & $-0,062$ & $3,136 * *$ & $-0,074$ & $2,095^{* *}$ & $-0,164$ & $1,961 * *$ & 0,006 & $1,678^{*}$ & 0,108 & $1,744 *$ \\
\hline Trabajadores manuales calificados & $0,613^{*}$ & $2,410 * *$ & $0,670 *$ & $1,187+$ & $0,610+$ & $1,174+$ & $0,658+$ & $1,197+$ & $0,758^{*}$ & $1,216+$ \\
\hline Trabajadores manuales no calificados & 0,404 & $2,189 * *$ & $0,513+$ & 0,828 & 0,421 & 0,739 & $0,575+$ & 0,722 & $0,576+$ & 0,729 \\
\hline Educación (en años) & - & - & 0,054 & $-0,176 * *$ & $0,093+$ & $-0,154 * *$ & $0,122 *$ & $-0,166 * *$ & $0,127 *$ & $-0,170 * *$ \\
\hline \multicolumn{11}{|l|}{ Tamaño de la localidad } \\
\hline Grande & - & - & $-0,199$ & $0,682 * *$ & $-0,276$ & $0,677 * *$ & $-0,256$ & $0,708^{*}$ & $-0,254$ & $0,687 * *$ \\
\hline Mediana & - & - & $-0,489 *$ & $0,309+$ & $-0,490 *$ & $0,481+$ & $-0,423+$ & $0,642^{*}$ & $-0,439+$ & $0,626 * *$ \\
\hline Ingreso & - & - & - & - & $-0,002$ & $-0,000 *$ & $-0,002$ & $0,000 * *$ & $-0,002$ & $0,008 * *$ \\
\hline Ingreso no declarado & - & - & - & - & $-0,282$ & $-0,292$ & $-0,292$ & $-0,117$ & $-0,262$ & $-0,087$ \\
\hline Activos hogar / Posición social & - & - & - & - & $-0,179 * *$ & $-0,031$ & $-0,196 * *$ & $-0,041$ & $-0,214^{* *}$ & $-0,042$ \\
\hline Condiciones crónicas & - & - & - & - & - & - & $2,163^{* *}$ & $2,314 * *$ & $1,922 * *$ & $2,272 * *$ \\
\hline Consultas al médico & - & - & - & - & - & - & - & - & $1,181 * *$ & 0,243 \\
\hline Constante & $-3,837 * *$ & $-5,330 * *$ & $-4,719 * *$ & $-2,401 * *$ & $-4,169 * *$ & $-2,157 * *$ & $-3,372 * *$ & $-2,350 *$ & $-3,565 * *$ & $-2,432 * *$ \\
\hline-2 log de la verosimilitud & 929,905 & 737,263 & 914,706 & 696,581 & 899,062 & 691,127 & 791,282 & 589,450 & 778,201 & 588,561 \\
\hline Número de casos & 1.191 & 865 & 1.184 & 865 & 1.184 & 865 & 1.184 & 865 & 1.184 & 865 \\
\hline
\end{tabular}

Nota: $* * \mathrm{p}<0,01 ; * \mathrm{p}<0,05 ;+\mathrm{p}<0,10$

Fuente: Elaboración propia en base a datos para Argentina de la Encuesta Nacional 2005, Centro de Estudios de Opinión Pública, Universidad de Buenos Aires (CEDOP-UBA) y Dirección de Estadísticas e Información en Salud, Ministerio de Salud de la Nación (DEIS-MSN); y datos para Japón de la Encuesta Ómnibus Nacional 2000, Central Research Services, Módulo Salud Agregado. 
En lo que hace a los efectos de la educación, Ishida nota que, contrario a sus expectativas, esta última tiene un efecto positivo sobre la percepción desfavorable de salud. En nuestro caso, los resultados coinciden con las expectativas iniciales de este autor, en el sentido de que la educación se asocia a una buena percepción de la propia salud. Por lo que respecta al tamaño de las localidades donde viven los encuestados, en el caso de Japón los que viven en ciudades intermedias tienden a percibir un mejor estado de salud, comparados con los que viven en pequeñas localidades o zonas rurales. A nivel local también la vida más urbana se asociaría a la mala percepción de salud. Aparentemente, distinto de Japón, también los menores ingresos se vincularían a identificar como desfavorable su propia salud en el nivel local. Se dice "aparentemente" porque la tenencia de activos en el hogar se asocia fuertemente con una buena percepción de salud en Japón. En cuanto al efecto de las condiciones de salud, en Japón se repite la presencia significativa tanto de condiciones crónicas como de visitas al médico, mientras que en Argentina solo la primera se asocia a la mala auto-percepción de salud. O sea, las visitas al médico, tomadas como indicador de accesibilidad, no se asocian en Argentina con la mala percepción de salud.

\section{CONCLUSIONES}

El uso de servicios de salud tiende a ser mayor en las clases más acomodadas y en sectores más altos de ingreso. Con la particularidad de que los usuarios de medicamentos exhiben una pauta limitada en este sentido, mientras que la consulta médica constituye el único tipo de utilización con una presencia ligeramente mayor en los sectores más pobres.

Respecto al gasto en salud, el promedio de gasto global en salud habría aumentado un $30 \%$ de 2003 a 2005. Ello no sería uniforme para todos los sectores: tiende a crecer el gasto en salud del sector más pobre, seguido por los sectores medios. En el sector más pobre el gasto en medicamentos es el que menos crece, creciendo más la afiliación voluntaria y la consulta médica. El promedio de gasto en consultas del sector de altos ingresos, en particular la consulta médica, es el único que cae en el bienio considerado.

En cuanto a los diferenciales sociales en salud, Ishida nota que la ausencia de efectos de factores socioeconómicos sobre condiciones crónicas y restricción de actividades es contraria a su hipótesis original. Esta situación a nivel local se da solo para la presencia de enfermedades crónicas, con cierta relevancia de los factores socioeconómicos. Puntualiza Ishida que:

\footnotetext{
...la emergencia de enfermedades crónicas y la subsiguiente limitación de actividades cotidianas son básicamente producidas por factores genéticos y constitucionales que pueden ser independientes de las diferencias socioeconómicas (8 p.18).
}

Si tal fuere el caso, esto es algo menos marcado en Argentina para la restricción de actividades. En general, Ishida observa que clase social y tenencia de activos son las condiciones socioeconómicas más relevantes, casi con independencia del ingreso y de los años de educación, para las condiciones de salud. En Argentina, se agrega la relevancia de la educación, además del ingreso para la salud auto-informada, sin importar la percepción de posición social (h). Y en ambos casos, las condiciones socioeconómicas persisten, aun controlando por condiciones de salud tales como enfermedades crónicas y visitas al médico.

Dada la importancia que la propuesta de Ishida otorga a la clase social, trata de especificar sus alcances. En el caso de las visitas al médico, la pequeña burguesía y las clases rurales tienden a visitar menos al médico que la clase de servicios (profesional-gerencial). Lo del sector rural puede deberse a la mayor dificultad de acceso, mientras que encuentra menos clara la poca concurrencia de la pequeño burguesía urbana. En cuanto a la auto-percepción de salud, serían los trabajadores manuales los que tienden a percibir peor su salud en Japón, comparados con la clase de servicios. Las condiciones más desfavorables del trabajo manual llevarían a mayores problemas físicos y a una peor percepción de salud.

Para el caso argentino, las clases rurales tienen alguna presencia en las enfermedades crónicas, comparando con la clase de servicios. 
Dejando de lado los trabajadores no manuales rutinarios, todas las otras clases, comparadas con la clase de servicios, exhiben menores chances de concurrencia al médico. Para la restricción de actividades, en una tendencia poco clara, la clase media de trabajadores no manuales rutinarios exhibe menores chances de restricciones que la clase de servicios. En lo que respecta a la mala percepción de su salud, todas las clases tienen más chances de una respuesta en ese sentido, comparadas con la clase en la cúspide, la clase profesional-gerencial. La conclusión de Ishida en este punto es válida para ambos relevamientos:

Todos estos hallazgos sugieren que las posiciones en el mercado de trabajo tienen una profunda influencia sobre la salud de los trabajadores (8 p.20).
De las hipótesis propuestas por Ishida, las que vinculan clase social medio-alta $y$ mayor educación con mejores condiciones de salud, se sostienen para Argentina (i). Con sus variaciones para cada país -fuera de las enfermedades crónicas muy condicionadas por la edad-, la clase importa. En cambio, la hipótesis que asociaba altos ingresos con buenas condiciones de salud se expresa de manera limitada, solo para el caso de la auto-percepción de salud en Argentina. Es decir: en presencia de la clase social, la vinculación entre ingresos y condiciones de salud -generalmente señalada en la bibliografía- no tiende a aparecer en las ecuaciones consideradas.

\section{NOTAS FINALES}

a. Ambas encuestas fueron realizadas gracias a becas otorgadas por la Comisión Nacional de Programas de Investigación Sanitaria (CONAPRIS), Ministerio de Salud de la Nación. El CEDOP-UBA se encargó del relevamiento. Los instrumentos y bases de datos pueden solicitarse a J. R. Jorrat. Agradecemos las muy importantes sugerencias $y$ observaciones de referatos anónimos de Salud Colectiva.

b. La afiliación se define como la integración de una parte de la población a alguna institución que brinda y/o financia servicios para la atención de su salud: consultas con el médico, consultas con el dentista, internaciones, análisis de laboratorio, etc. La afiliación a "obra social" refiere a la cobertura de salud que obtienen las personas que trabajan y sus familiares mediante afiliación obligatoria, incluyendo la cobertura legal que reciben las personas jubiladas o pensionadas. La afiliación a "plan de salud privado o mutual" es una modalidad de aseguramiento de la salud, caracterizada por la adhesión voluntaria y el pago del servicio por parte del beneficiario en su totalidad. La no afiliación supone que las personas de esta condición sólo tienen la cobertura del Subsector Público de Atención de la Salud. 
c. La encuesta descansa en una muestra estratificada por áreas -según Necesidades Básicas Insatisfechas (NBI) a nivel de radio censal-, multietápica, con selección aleatoria en todas las etapas salvo la selección final del encuestado, realizada según cuotas de sexo y edad. Los estratos especificados fueron 26, de acuerdo al tamaño de las localidades, incluyendo población rural agrupada y dispersa. Para un tamaño inicial de 1.000 adultos (18 años y más), se agregaba, en cada hogar donde se encuestaba un adulto, un menor (de 0 a menos de 18 años) que era seleccionado de acuerdo al cumpleaños más reciente. Ello arrojó un total muestral de 1.546 casos, personas de todas las edades (0 años y más). Para aquellos entre 0 y menos de 18 años, respondía la madre, u otro adulto en caso de no existir o no acceder a la madre. Bajo el supuesto de muestreo aleatorio simple de 1.546 casos, el error muestral, con un nivel de confianza del $95 \%$, si se trabajase con datos que sumen el total muestral, sería de +/$2,5 \%$. De todas formas, de acuerdo a normas de la DEIS-MSN se trabaja con datos expandidos a la población, según el Censo Nacional de 2001, lo que se respetó en esta presentación, salvo en el caso de las regresiones para la comparación con Japón. Tomando en cuenta el valor relativo o coeficiente de variabilidad, valores inferiores a 570.000 casos deberían considerarse con precaución. La tasa de respuesta estaría, aproximadamente, en un 50\%. El trabajo de campo se extendió de septiembre a diciembre de 2005.

d. ENFERMEDADES CRÓNICAS: ¿Alguna vez un médico le diagnosticó a usted la presencia de una enfermedad que se extiende en el tiempo y que requiere tratamiento prolongado, como las que se mencionan a continuación? CONSULTAS (VISITAS) AL MÉDICO: Durante los últimos 30 días, ¿hizo consultas con el médico, como clínicos, cirujanos, ginecólogos, oculistas, etc.? NO-Sí; si "SÍ": ¿Cuántas consultas hizo en total? [Para los no consultantes de los últimos 30 días, se interroga sobre el tiempo que hace que consultó al médico. Los consultantes dentro del último año se tomaron como que visitaron al médico]. RESTRICCIÓN ACTIVIDADES: ¿Ha dejado usted de hacer alguna de las siguientes actividades por problemas de salud en los últimos 12 meses? a) Ir a su trabajo; b) Hacer trabajos en la casa; c) Ir a la escuela, colegio o universidad. [La respuesta afirmativa a solo una indicaba restricción]. AUTOPERCEPCIÓN DE SALUD: ¿Diría usted, en líneas generales, que su estado de salud es...? 1. Excelente; 2. Muy bueno; 3. Bueno; 4. Regular; 5. Malo; 6. Muy malo. [Se codificaba 1 -como no buen estado- si decían regular, malo o muy malo, 0 en otro caso].

e. No se ofrecen pruebas de diferencias de medias. Los promedios están calculados con datos expandidos al total poblacional (personas de 0 años y más), por lo que sería imposible que una diferencia, por pequeña que fuere, no resulte estadísticamente significativa. Si se realizaran -previas a la expansión- para todos los cortes sociodemográficos de los promedios de cada gasto, implicaría una larga y engorrosa presentación (A versus B, A versus $C$, etc.) Para un intento breve más de tipo descriptivo que inferencial como en este caso, nos parece conveniente evitar una presentación complicada, fuera de recordar las discusiones sobre algunos excesos en la consideración de la significación estadística (15).

f. Ishida señala que "no es claro por qué la pequeña burguesía urbana exhibe menores chances de visitar al médico. La interpretación más probable yace en su largo horario de trabajo. [...] La pequeña burguesía urbana puede no ser capaz de hacerse tiempo para visitar al médico" (8 p.13).

g. Estos valores surgen del exponencial (b) -correspondiente al coeficiente de regresión logística binaria b-, en las ecuaciones de regresión logística. Estos valores no fueron presentados en los cuadros de Ishida, pauta que seguimos a efectos comparativos. Dichos valores los comenta Ishida en el texto.

h. Lamentablemente, Ishida no hace referencias a los posibles problemas de altas correlaciones entre activos del hogar e ingresos (multicolinealidad), que podrían afectar la presencia conjunta de estas dos variables en la ecuación.

i. Más allá de las diferencias de enfoques y de medición de las variables, si se elimina clase social y se considera una regresión logística con visitas al médico o al dentista en el último mes -para poder comparar- como variable dependiente y sexo 
(varón $=1$ ), edad, años de educación, ingresos per cápita del hogar, ingresos no declarados $(=1$ si no se declara), afiliación ( $=1$ si es afiliado), tamaño de localidad (como en el texto, usando como referencia las pequeñas y rurales) y nivel de hacinamiento (=1 si 3 o más personas por cuarto), los resultados tienden a coincidir con un estudio basado en una base de datos mucho más amplia, unos 20.000 casos relevados por la Encuesta de Condiciones de Vida (ECV) del Sistema de Información, Monitoreo y Evaluación de Programas Sociales (SIEMPRO) en 2001: "La probabilidad de consultar a los profesionales de la salud depende positivamente del ingreso per capita familiar, de residir en las regiones más desarrolladas del país, de poseer cobertura de salud, del nivel de educación del individuo y disminuye con el nivel de hacinamiento del hogar del individuo" (19 p.17). Si bien es otro el análisis -no usan regresiones logísticas- las similitudes son de gran interés. Una diferencia es que se agrega en nuestro estudio la mayor probabilidad de visitas si se es mujer, no siendo significativo el tamaño de la localidad (en el otro estudio se consideraron regiones). Tendencias en esta línea, para los mismos datos, parecen surgir de un estudio que considera la salud auto-informada (20).

\section{REFERENCIAS BIBLIOGRÁFICAS}

1. Jorrat JR, Fernández MM, Marconi E. Utilización y gasto en servicios de salud y medicamentos. En: Tobar F, Godoy Garraza L, coordinadores. El futuro del acceso a los medicamentos en la Argentina. Buenos Aires: Consejo Nacional de Programas de Investigación en Salud, Ministerio de Salud; 2004. p. 58-76.

2. Zimmer Z, Natividad J, Lin WH, Chayovan N. A Cross-national Examination of the Determinants of Self-Assed Health. Journal of Health and Social Behavior. 2000;41(4):465-481.

3. Bertranou FM. Desigualdades en salud asociadas al ingreso: evidencia empírica para la Argentina. Córdoba: Universidad Siglo XXI; 2001. [Documento de Investigación].

4. Mackenbach JP, Kunst AE. Measuring the Magnitude of Socio-Economic Inequalities in Health: An Overview of Available Measures Illustrated with Two Examples from Europe. Social Science and Medicine. 1997;44(6):757-771.
5. Mechanic D. The Role of Sociology in Health Affairs. Health Affairs. 1990;9(1):85-97.

6. Light DW. Introduction: Strengthening Ties between Specialties and the Discipline. The American Journal of Sociology. 1992;97(4): 909-918.

7. Rodríguez JA, De Miguel JM. Salud y Poder. Madrid: CIS; 1990.

8. Ishida $\mathrm{H}$. Socio-economic differentials in Health in Japan. [En línea]. Ponencia presentada al International Sociological Association's Research Committee on Social Stratification (RC28); 7 al 8 de agosto de 2004; Río de Janeiro, Brasil. Río de Janeiro: Instituto Universitário de Pesquisas do Rio de Janeiro (IUPERJ) [fecha de acceso 20 de abril de 2007]. URL disponible en: http://www.iuperj.br/rc28/papers/Health704sentwithtab1hisroshiishida.pdf

9. Gasparini LC. On the measurement of unfairness. An application to high school attendance in Argentina. Social Choice and Welfare. 2002;9(4):795-810. 
10. Kawachi I, Subramanian SV, Almeida-Filho N. A glossary for health inequalities. Journal of Epidemiology and Community Health. 2002;56(9):647-652.

11. Gwatkin DR. Health inequalities and the health of the poor: What do we know? What can we do? (Critical Reflection). Bulletin of the World Health Organization. 2000;78(1):3-18.

12. Acheson D. Round Table Discussion. Health inequalities impact assessment. Bulletin of the World Health Organization. 2000;78(1):74-75

13. Programa Nacional de Estadísticas de Salud. Encuesta a población. Serie 10, № 17. Buenos Aires: DEIS-MSN; 2004.

14. Erikson R, Goldthorpe JH, Portocarero, L. Intergenerational Class Mobility in Three Western European Societies. British Journal of Sociology. 1979;30(4):415-441.

15. Erikson R, Goldthorpe JH. The Constant Flux: A Study of Class Mobility in Industrial Nations. Oxford: Clarendon Press; 1992.
16. Jorrat JR. Estratificación social y movilidad. Un estudio del área metropolitana de Buenos Aires. Tucumán: EUdeT; 2000.

17. Morrison D, Henckel R. The Significance Test Controversy. New Jersey: Aldaine; 2006.

18. Pampel FC. Logistic Regression. A Primer. Thousand Oaks (California): Sage; 2000.

19. De Santis M, Herrero V. Equidad en el acceso, desigualdad y utilización de los servicios de salud. Una aplicación al caso argentino en 2001. [En línea]. Asociación Argentina de Economía Política. XLI Reunión Anual. Salta: UNSa, UCS; 2006. [Fecha de acceso 28 de mayo de 2007]. URL disponible en: http://www.aaep.org.ar/espa/anales/works06/D e_Santis_Herrero.pdf

20. De Maio F. Health inequalities in Argentina: patterns, contradictions, and implications. Health Sociology Review. 2007;16(3-4):279-291.

\section{BIBLIOGRAFÍA DE CONSULTA}

Tobar F, coordinador. El Gasto en Salud en Argentina y su Método. [En línea] Buenos Aires: Instituto Universitario, Programa de Investigación Aplicada; 2000 [fecha de acceso 15 de mayo de 2007]. URL disponible en: http://www.who.int/nha/docs/es/Argentina_NHA _report_spanish.pdf

\section{FORMA DE CITAR}

Jorrat JR, Fernández MM, Marconi EH. Utilización y gasto en servicios de salud de los individuos en Argentina en 2005. Comparaciones internacionales de diferenciales socio-económicos en salud. Salud Colectiva. 2008;4(1):57-76.

Recibido el 2 de julio de 2007

Versión final presentada el 16 de noviembre de 2007

Aprobado el 27 de diciembre de 2007 